\title{
COVID-19: Impacto Psicológico, Factores de Riesgo e Intervenciones Psicológicas en el Personal Sanitario. Una Revisión Sistemática.
}

\begin{abstract}
María Dolores Tolsa ${ }^{\star}$ y Olga Malas ${ }^{2}$.
' Departamento de Medicina Experimental. Universitat de Lleida (UdL)

2 Servicios Integrales de Psicología. Emergencias Médicas de Catalunya (SEM)

. Recibido: 19 - 09 - 2020 . Aceptado: 24 - 03 - 2021 . Avance online: 10 - 04 - 2021

RESUMEN. En relación a la mala salud mental del personal sanitario debido a la pandemia por COVID-19, la presente revisión sistemática tiene como objetivo sintetizar el conocimiento generado entre noviembre-2019 y septiembre-2020 sobre impacto psicológico, factores de riesgo e intervenciones psicológicas llevadas a cabo en esta población. Siguiendo metodología PRISMA se recuperaron 49 artículos que cumplieron con todos los criterios de inclusión y calidad exigidos, y que revelaron prevalencias del 5,2\% al 79\% para estrés, del 6,78\% al $71 \%$ para los trastornos del sueño, del 4,98\% al 50\% para ansiedad, del 8,9\% al 50,70\% para depresión y del 4,4\% al 19,10\% para estrés postraumático. Las mayores prevalencias dependen de factores relacionados con situaciones de mayor riesgo (primeras fases de la pandemia, primera línea, escasez de recursos de protección personal y falta de apoyo social) y con la pertenencia a grupos poblacionales más susceptibles (mujeres, personal sanitario de apoyo, con poca formación sobre la COVID-19, de nivel socioeconómico bajo y/o vida sedentaria, entre otros). Prevalecen las intervenciones basadas en estrategias de intervención temprana, de resolución de problemas inmediatos y terapias cognitivo-conductual para incrementar las conductas adaptativas, mejorar las habilidades de afrontamiento al estrés y establecer sistemas de apoyo social.
\end{abstract}

PALABRAS CLAVE: COVID-19, Personal sanitario, Impacto psicológico, Factores de riesgo, Intervención psicológica.

COVID-19: Psychological Impact, Risk Factors and Psychological Interventions in Healthcare Personnel.

A Systematic Review.

ABSTRACT. Relating to poor mental health of healthcare workers due to COVID-19 pandemic, the present systematic review aims to synthesize the knowledge generated between November-2019 and September-2020 about psychological impact, risk factors and psychological interventions carried out on this population. Following PRISMA methodology, we retrieved 49 titles, which fulfilled all inclusion and quality criteria; and that revealed prevalences ranging from $5.2 \%$ to $79 \%$ for stress, from $6.78 \%$ to $71 \%$ for sleep disturbances, from $4.98 \%$ to $50 \%$ for anxiety, from $8.9 \%$ to $50.70 \%$ for depression and from $4.4 \%$ to $19.10 \%$ for post-traumatic stress disorder. Higher prevalences were dependent on experiencing situations of increased risk (early stages of the pandemic, working on the front line, the scarcity of personal protection resources or the lack of social support) and the membership to susceptible population groups (being a woman, working as a health support staff, have little training on COVID-19, being of low socioeconomic status and/or have a sedentary lifestyle among others). Prevail the interventions based on early intervention strategies, for solving immediate problems, and cognitivebehavioral therapy in order to increase adaptive behaviors, greater coping skills to stress, and establish social support systems.

KEYWORDS: COVID-19, Health personnel, Psychological impact, Risk factors, Psychological intervention.

La pandemia por coronavirus, conocida como COVID-19, puede catalogarse como la mayor crisis sanitaria del mundo de las últimas

\footnotetext{
*Correspondencia: María Dolores Tolsa

Departamento de Medicina Experimental. Universitat de Lleida (UdL)

Dirección: 25006. Lleida, España.

E-mail: mariadolores.tolsa@udl.cat
}

décadas. La rapidez de implantación y la alta prevalencia ha colapsado hospitales y otros servicios de salud, sometiendo al personal sanitario a una presión psicológica considerable capaz de originarles problemas de salud mental como ansiedad o depresión (Chew et al., 2020; Guo et al., 2020) y pudiendo afectar a su capacidad para controlar la enfermedad (Chen et al., 2020a). Por ello, muchos investigadores 
han trabajado para establecer el impacto psicológico y los factores de riesgo asociados, haciendo propuestas de intervención para prevenir y ayudar a los afectados.

Esto ha generado una gran cantidad de información que debe ser analizada y consolidada para que los resultados sean más manejables para su consulta. Hemos encontrado 3 revisiones sistemáticas previas. Dos en población general. La primera, efectuada por Rajkumar (2020), incluyó 28 artículos, publicados antes de abril de 2020. La segunda fue realizada por Luo, Guo, Yu y Wang (2020) tras seleccionar 62 artículos publicados entre noviembre de 2019 y el 25 de mayo de 2020. En personal sanitario solo hemos encontrado, la realizada por Pappa et al. (2020), para establecer la prevalencia de depresión y ansiedad en esta población, utilizando 13 artículos publicados antes del 17 de abril de 2020.

En este contexto, nos planteamos hacer una nueva revisión sistemática ampliando la búsqueda hasta septiembre de 2020, con el objetivo de actualizar los datos existentes sobre el impacto psicológico, los factores de riesgo y las intervenciones psicológicas en personal sanitario. El objetivo último de este documento es describir la prevalencia y tipología de los problemas de salud mental entre el personal sanitario durante la epidemia de COVID-19, identificar los factores de riesgo y la población clave de intervención psicológica y analizar los planes de intervención llevados a cabo.

\section{MÉTODO}

\section{- DISEÑO Y PROCEDIMIENTO}

Para seleccionar los artículos se ha seguido metodología PRISMA (Liberaty et al., 2009). Para la recogida de información se creó un protocolo de revisión y se utilizó la plantilla TIDieR (Hoffmann et al., 2016). Este protocolo fue aplicado en paralelo por dos investigadores independientes. La información discordante se discutió y consolidó resolviendo los desacuerdos mediante discusión y consenso.

Para la búsqueda de los artículos se revisó inicialmente la información para COVID-19 disponible en la web Cochrane Collabotarion y en la OMS (Organización Mundial de la Salud). Seguidamente se realizó una búsqueda sistemática, desde noviembre de 2019 hasta la primera semana de septiembre de 2020, utilizando los términos de búsqueda "COVID-19" AND "Psicología" AND "Personal Sanitario", en español, inglés, francés, italiano y portugués en las bases de datos Scopus, Psyclnfo, PsycArticles y Google Académico.

Puesto que no existe un método estandarizado para medir los factores psicológicos y a causa de la gran variabilidad hallada entre estudios (tipo de muestra, fase de pandemia, instrumentos de medición, etc.), el protocolo no incluyo el meta-análisis, centrándose en una revisión sistemática más narrativa que cuantitativa.

\section{- CRITERIOS DE ELEGIBILIDAD}

\section{CRITERIOS DE INCLUSIÓN}

Para la inclusión de los artículos, se eligieron investigaciones originales prospectivas efectuadas sobre personal sanitario o investigaciones en población general, siempre que incluyeran muestras en personal sanitario y datos comparativos entre poblaciones. Teniendo en cuenta la rápida difusión de información durante la pandemia, se aceptaron artículos preimpresos.

\section{CRITERIOS DE EXCLUSIÓN}

Fueron criterios de exclusión no cumplir con alguno de los criterios de inclusión establecidos, la baja calidad metodológica (descripción inadecuada de la muestra, de la fecha de estudio, de la metodología seguida o de los resultados); o estar en un idioma diferente al inglés, español, alemán, francés, italiano y portugués.

\section{- RIESGO DE SESGO: CALIDAD METODOLÓGICA}

La calidad metodológica de los estudios se evaluó mediante la herramienta RoB2 de Cochrane Risk of Bias (Higgins et al., 2019). En todo caso, el riesgo de sesgo es significativo en 
todos los estudios analizados, por lo que solo fueron descartados los artículos con alto riesgo de sesgo.

\section{- FIABILIDAD DE LA CODIFICACIÓN}

La codificación de los artículos fue efectuada por los dos investigadores de forma independiente, a dos tiempos, de forma similar a Monteiro, Vázquez, Seijo y Arce (2018) y Álvarez-Muelas, Gómez-Berrocal y Sierra (2020), siguiendo las recomendaciones de ajuste indicadas en Cochrane Risk of Bias (Higgins et al., 2019), la concordancia se hizo determinando el estadístico kappa verdadera $(\bar{\kappa}>0,80)$.

Siguiendo la técnica de análisis de contenido categorial y aplicando la solución estadística de Fariña, Arce y Novo (2002), la codificación fue evaluada en términos de acuerdo intercodificadores e intra-codificadores con la kappa verdadera $(\bar{\kappa})$. Para ello se registró la codificación como 1 acuerdo si la codificación entre investigadores y diferentes tiempos era la misma;
1 desacuerdo si un codificador, o en un momento, registraba una categoría y otro codificador, o en otro momento, no la registraba; y 2 desacuerdos si ambos codificadores, o en ambos momentos, registraban la misma categoría, pero de modo diferente. Los resultados de la concordancia verdadera mostraron una concordancia intere intra-evaluador en todas las categorías muy buena $(\bar{\kappa}>0,87)$. Ello permite concluir que cotejada la consistencia inter- e intra-evaluador e inter-contextos, la codificación es fiable y que otro codificador entrenado en la técnica de codificación hubiera creado un base de datos similar (Monteiro, Vázquez, Seijo y Arce, 2018).

\section{RESULTADOS}

Siguiendo la metodología PRISMA (ver flujo de selección y motivos de descarte en Figura 1), se seleccionaron 49 artículos, 46 fueron usados para analizar el impacto y los factores de riesgo psicológicos y 7 para analizar las intervenciones psicológicas.

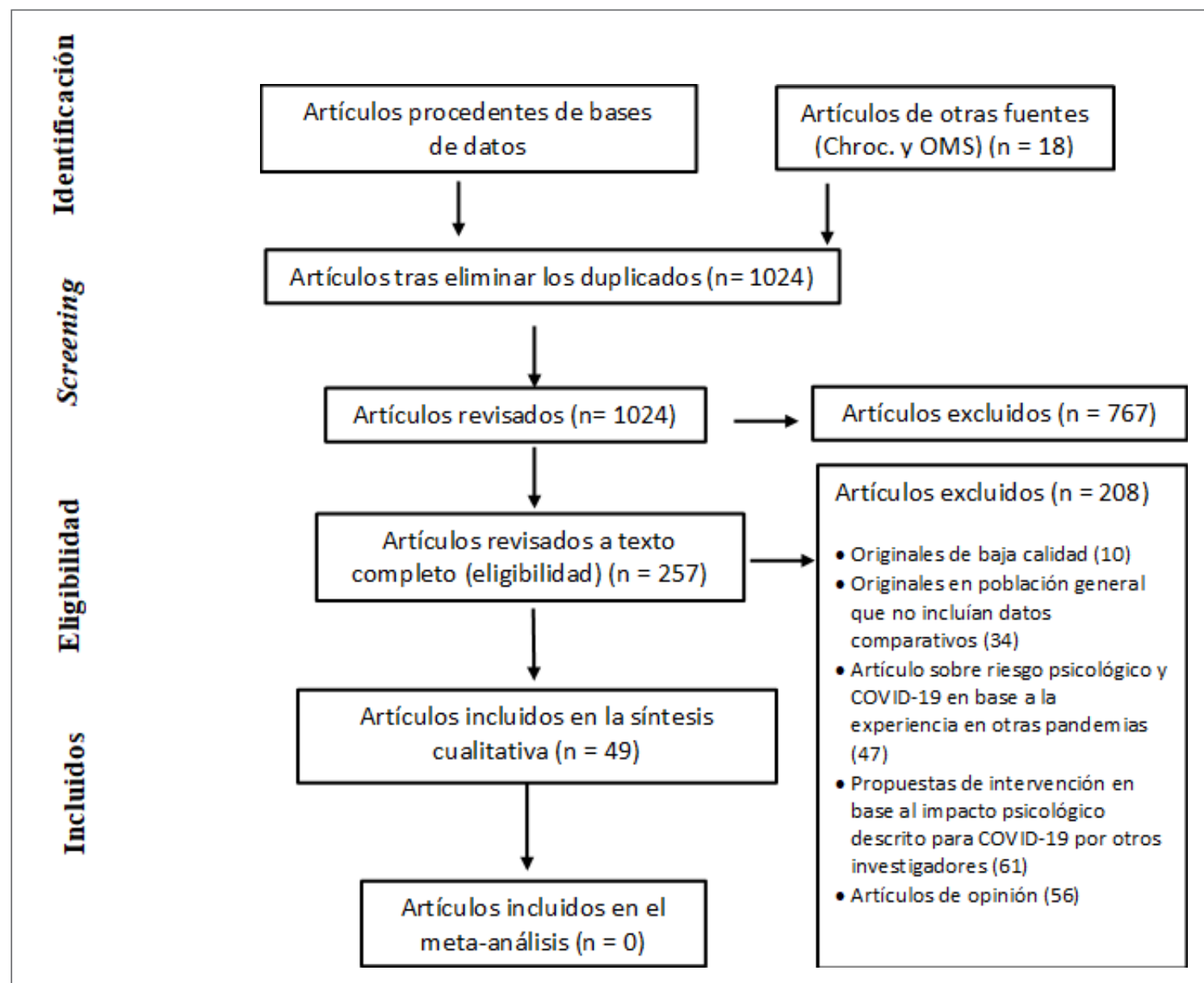

Figura-7: Búsqueda y selección de estudios 


\section{- IMPACTO PSICOLÓGICO Y FACTORES DE RIESGO}

Los resultados obtenidos para impacto psicológico y factores de riesgo pueden verse en Tabla 1. Los datos de prevalencia recopilados muestran resultados muy variables, pudiendo establecer horquillas que oscilan de 5.2\% a $79 \%$ para estrés, de $6.78 \%$ a $71 \%$ para alteraciones del sueño, de $4.98 \%$ a $50 \%$ para ansiedad, de $8.9 \%$ a $50.70 \%$ para depresión y de $4.4 \%$ a $19.10 \%$ para estrés postraumático. Esta variabilidad podría estar relacionada con la fase de la pandemia y los factores de riesgo asociados.

Tabla 7

Impacto psicológico y factores de riesgo del COVID-19 en personal sanitario

\begin{tabular}{|c|c|c|c|c|c|c|}
\hline Referencias & Muestra & Origen & Método & Instrumentos* & $\begin{array}{c}\text { Variables } \\
\text { analizadas } \\
\text { y resultados } \\
\text { principales }\end{array}$ & $\begin{array}{c}\text { Factores de riesgo } \\
\text { identificados }\end{array}$ \\
\hline $\begin{array}{l}\text { Abdessater et } \\
\text { al. (2020) }\end{array}$ & $\begin{array}{c}\text { Personal sanitario } \\
(\mathrm{n}=275) \\
\text { Edad media: } \\
29,5 \pm 0,27 \\
\text { Género femenino: } \\
32,7 \%\end{array}$ & Francia & $\begin{array}{l}\text { Encuesta } \\
\text { transversal } \\
\text { en línea } \\
\text { (ETL) }\end{array}$ & $\mathrm{MBI}$ & $\begin{array}{c}\text { El } 56,5 \% \text { de la muestra } \\
\text { presenta elevados } \\
\text { niveles de estrés. }\end{array}$ & $\begin{array}{l}\text { Falta de formación. No } \\
\text { contar con directrices } \\
\text { claras para trabajar. No } \\
\text { contar con equipos de } \\
\text { protección adecuados. }\end{array}$ \\
\hline $\begin{array}{l}\text { Ashtari et al. } \\
\text { (2020) }\end{array}$ & $\begin{array}{c}\text { Población general } \\
\text { ( } \mathrm{n}=886 \text { ) incluyendo } \\
\text { personal sanitario } \\
(\mathrm{n}=217) \\
\text { Edad media: } \\
40,9 \pm 10,7 \\
\text { Género femenino: } \\
37,5 \%\end{array}$ & Irán & ETL & DASS-21 & $\begin{array}{l}\text { Los puntajes medios } \\
\text { en depresión, } \\
\text { ansiedad y estrés } \\
\text { son más altos en } \\
\text { estudiantes de } \\
\text { medicina y pacientes } \\
\text { con COVID-19 que } \\
\text { en personal médico y } \\
\text { otras poblaciones. }\end{array}$ & Niguno \\
\hline $\begin{array}{l}\text { Cai et al. } \\
\text { (2020a) }\end{array}$ & $\begin{array}{c}\text { Personal sanitario } \\
\quad(\mathrm{n}=534) \\
\text { Rango de edad: } \\
18-40 \\
\text { Género femenino: } \\
68,73 \%\end{array}$ & China & ETL & QP & $\begin{array}{l}\text { El } 74,9 \% \text { de la muestra } \\
\text { presenta elevados } \\
\text { niveles de estrés. }\end{array}$ & $\begin{array}{l}\text { Falta de equipos de } \\
\text { protección adecuados. } \\
\text { Agotamiento por exceso } \\
\text { de trabajo. Preocupación } \\
\text { por los pacientes al } \\
\text { no poder atenderlos } \\
\text { adecuadamente. }\end{array}$ \\
\hline $\begin{array}{l}\text { Cai et al. } \\
\text { (2020b) }\end{array}$ & $\begin{array}{l}\text { Personal sanitario } \\
(n=1.521) \\
\text { Género femenino: } \\
75,54 \%\end{array}$ & China & ETL & $\begin{array}{c}\text { CD-RISC, SSRS Y } \\
\text { SCL-90-R }\end{array}$ & $\begin{array}{c}\text { El personal sanitario sin } \\
\text { experiencia presenta } \\
\text { niveles más elevados } \\
\text { de estrés y ansiedad } \\
\text { fóbica. }\end{array}$ & $\begin{array}{l}\text { Falta de experiencia } \\
\text { cuidando pacientes con } \\
\text { COVID-19. Preocupación } \\
\text { por transmitir la } \\
\text { enfermedad a su familia. }\end{array}$ \\
\hline $\begin{array}{l}\text { Cao et al. } \\
\text { (2020) }\end{array}$ & 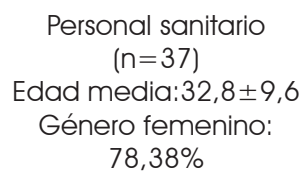 & China & ETL & MBI y $\mathrm{PHQ}-9$ & $\begin{array}{c}\text { El } 29,7 \% \text { de la muestra } \\
\text { presenta alteraciones } \\
\text { del sueño. }\end{array}$ & Ninguno \\
\hline $\begin{array}{l}\text { Chen, Zhuo, } \\
\text { Zhuo y Zhuo } \\
\text { (2020b) }\end{array}$ & $\begin{array}{c}\text { Personal sanitario } \\
(\mathrm{n}=105) \\
\text { Edad media: } \\
32,6 \pm 6,5 \\
\text { Género femenino: } \\
90,48 \%\end{array}$ & China & ETL & SAS y SDS & $\begin{array}{l}\text { El 18,1\% de la muestra } \\
\text { presenta niveles altos } \\
\text { de ansiedad y el } \\
29,5 \% \text { de depresión. }\end{array}$ & $\begin{array}{c}\text { Falta de experiencia } \\
\text { cuidando pacientes } \\
\text { con COVID-19. Tener } \\
\text { síntomas compatibles } \\
\text { con COVID-19. }\end{array}$ \\
\hline $\begin{array}{l}\text { Chew et al. } \\
(2020)\end{array}$ & $\begin{array}{c}\text { Personal sanitario } \\
\text { ( } n=906) \\
\text { Rango de edad: } 25 \\
\text { a } 30 \\
\text { Género femenino: } \\
64,36 \%\end{array}$ & $\begin{array}{l}\text { Singapur } \\
\text { e India }\end{array}$ & ETL & $\begin{array}{l}\text { DASS-21 y } \\
\text { IES-R }\end{array}$ & $\begin{array}{c}\text { El } 5,2 \% \text { de la muestra } \\
\text { presenta niveles altos } \\
\text { de estrés, el } 15,7 \% \\
\text { de ansiedad, el } \\
10,6 \% \text {, de depresión } \\
\text { y el } 7,4 \% \text { de Estrés } \\
\text { Postraumático. }\end{array}$ & Ninguno \\
\hline
\end{tabular}


Tabla 1 (Continuación)

Impacto psicológico y factores de riesgo del COVID-19 en personal sanitario

\begin{tabular}{|c|c|c|c|c|c|c|}
\hline $\begin{array}{l}\text { Abdessater et } \\
\text { al. (2020) }\end{array}$ & $\begin{array}{c}\text { Personal sanitario } \\
(n=275) \\
\text { Edad media: } \\
29,5 \pm 0,27 \\
\text { Género femenino: } \\
32,7 \%\end{array}$ & Francia & $\begin{array}{l}\text { Encuesta } \\
\text { transversal } \\
\text { en línea } \\
\text { (ETL) }\end{array}$ & $\mathrm{MBI}$ & $\begin{array}{l}\text { El } 56,5 \% \text { de la muestra } \\
\text { presenta elevados } \\
\text { niveles de estrés. }\end{array}$ & $\begin{array}{l}\text { Falta de formación. No } \\
\text { contar con directrices } \\
\text { claras para trabajar. No } \\
\text { contar con equipos de } \\
\text { protección adecuados. }\end{array}$ \\
\hline $\begin{array}{l}\text { Ashtari et al. } \\
\text { (2020) }\end{array}$ & $\begin{array}{c}\text { Población general } \\
\text { ( } \mathrm{n}=886 \text { ) incluyendo } \\
\text { personal sanitario } \\
(\mathrm{n}=217) \\
\text { Edad media: } \\
40,9 \pm 10,7 \\
\text { Género femenino: } \\
37,5 \%\end{array}$ & Irán & ETL & DASS-21 & $\begin{array}{l}\text { Los puntajes medios } \\
\text { en depresión, } \\
\text { ansiedad y estrés } \\
\text { son más altos en } \\
\text { estudiantes de } \\
\text { medicina y pacientes } \\
\text { con COVID-19 que } \\
\text { en personal médico y } \\
\text { otras poblaciones. }\end{array}$ & Niguno \\
\hline $\begin{array}{l}\text { Cai et al. } \\
\text { (2020a) }\end{array}$ & $\begin{array}{c}\text { Personal sanitario } \\
(n=534) \\
\text { Rango de edad: } \\
18-40 \\
\text { Género femenino: } \\
68,73 \%\end{array}$ & China & ETL & QP & $\begin{array}{l}\text { El } 74,9 \% \text { de la muestra } \\
\text { presenta elevados } \\
\text { niveles de estrés. }\end{array}$ & $\begin{array}{l}\text { Falta de equipos de } \\
\text { protección adecuados. } \\
\text { Agotamiento por exceso } \\
\text { de trabajo. Preocupación } \\
\text { por los pacientes al } \\
\text { no poder atenderlos } \\
\text { adecuadamente. }\end{array}$ \\
\hline $\begin{array}{l}\text { Cai et al. } \\
\text { (2020b) }\end{array}$ & $\begin{array}{c}\text { Personal sanitario } \\
(n=1.521) \\
\text { Género femenino: } \\
75,54 \%\end{array}$ & China & ETL & $\begin{array}{c}\text { CD-RISC, SSRS Y } \\
\text { SCL-90-R }\end{array}$ & $\begin{array}{c}\text { El personal sanitario sin } \\
\text { experiencia presenta } \\
\text { niveles más elevados } \\
\text { de estrés y ansiedad } \\
\text { fóbica. }\end{array}$ & $\begin{array}{c}\text { Falta de experiencia } \\
\text { cuidando pacientes con } \\
\text { COVID-19. Preocupación } \\
\text { por transmitir la } \\
\text { enfermedad a su familia. }\end{array}$ \\
\hline $\begin{array}{l}\text { Cao et al. } \\
\text { (2020) }\end{array}$ & $\begin{array}{c}\text { Personal sanitario } \\
(n=37) \\
\text { Edad media:32,8 } 89,6 \\
\text { Género femenino: } \\
78,38 \%\end{array}$ & China & ETL & MBI y $\mathrm{PHQ}-9$ & $\begin{array}{c}\text { El } 29,7 \% \text { de la muestra } \\
\text { presenta alteraciones } \\
\text { del sueño. }\end{array}$ & Ninguno \\
\hline $\begin{array}{l}\text { Chen, Zhuo, } \\
\text { Zhuo y Zhuo } \\
\text { (2020b) }\end{array}$ & $\begin{array}{c}\text { Personal sanitario } \\
(\mathrm{n}=105) \\
\text { Edad media: } \\
32,6 \pm 6,5 \\
\text { Género femenino: } \\
90,48 \%\end{array}$ & China & ETL & SAS y SDS & $\begin{array}{c}\text { El } 18,1 \% \text { de la muestra } \\
\text { presenta niveles altos } \\
\text { de ansiedad y el } \\
29,5 \% \text { de depresión. }\end{array}$ & $\begin{array}{c}\text { Falta de experiencia } \\
\text { cuidando pacientes } \\
\text { con COVID-19. Tener } \\
\text { síntomas compatibles } \\
\text { con COVID-19. }\end{array}$ \\
\hline $\begin{array}{l}\text { Chew et al. } \\
(2020)\end{array}$ & $\begin{array}{c}\text { Personal sanitario } \\
\quad(n=906) \\
\text { Rango de edad: } 25 \\
\text { a } 30 \\
\text { Género femenino: } \\
64,36 \%\end{array}$ & $\begin{array}{l}\text { Singapur } \\
\text { e India }\end{array}$ & ETL & $\begin{array}{l}\text { DASS- } 21 \text { y } \\
\text { IES-R }\end{array}$ & $\begin{array}{c}\text { El } 5,2 \% \text { de la muestra } \\
\text { presenta niveles altos } \\
\text { de estrés, el } 15,7 \% \\
\text { de ansiedad, el } \\
10,6 \% \text {, de depresión } \\
\text { y el } 7,4 \% \text { de Estrés } \\
\text { Postraumático. }\end{array}$ & Ninguno \\
\hline $\begin{array}{c}\text { Chung y Yeung } \\
\text { (2020) }\end{array}$ & Personal sanitario $=60$ & Canadá & ETL & PHQ-9 & $\begin{array}{l}\text { El } 29 \% \text { de la muestra } \\
\text { presenta elevados } \\
\text { niveles de estrés. }\end{array}$ & Ninguno \\
\hline $\begin{array}{c}\text { Consolo, } \\
\text { Bellini, } \\
\text { Bencivenni, } \\
\text { lani y Checchi } \\
\text { (2020) }\end{array}$ & $\begin{array}{l}\text { Personal sanitario } \\
\quad(n=356)\end{array}$ & Italia & ETL & GAD-7 & $\begin{array}{c}\text { El } 70,2 \% \text { de la muestra } \\
\text { presenta elevados } \\
\text { niveles de estrés y el } \\
46,4 \% \text { de ansiedad. }\end{array}$ & Ninguno \\
\hline Du et al. (2020) & $\begin{array}{c}\text { Personal sanitario } \\
(n=134) \\
\text { Edad media: } 36 \pm 8,05 \\
\text { Género femenino: } \\
60,45 \%\end{array}$ & China & ETL & BAI, BDI y PSS & $\begin{array}{l}\text { El } 59 \% \text { de la muestra } \\
\text { presenta elevados } \\
\text { niveles de estrés; el } \\
20,1 \% \text { de ansiedad; } \\
\text { y el } 12,7 \% \text { de } \\
\text { depresión. }\end{array}$ & $\begin{array}{c}\text { Ser mujer. Tener } \\
\text { poco apoyo familiar. } \\
\text { Preocupación y sensación } \\
\text { de incapacidad } \\
\text { para cuidar a los } \\
\text { adecuadamente. Mala } \\
\text { calidad del sueño y } \\
\text { descanso inadecuado. } \\
\text { Falta de formación para } \\
\text { afrontar el impacto } \\
\text { psicológico. }\end{array}$ \\
\hline
\end{tabular}


Tabla 1 (Continuación)

Impacto psicológico y factores de riesgo del COVID-19 en personal sanitario

\begin{tabular}{|c|c|c|c|c|c|c|}
\hline $\begin{array}{l}\text { Fu et al. } \\
\text { (2020) }\end{array}$ & $\begin{array}{c}\text { Población general } \\
\text { (n=1.242) incluyendo } \\
\text { personal sanitario } \\
(n=454) \text {. } \\
\text { Género femenino: } \\
69,7 \%\end{array}$ & China & ETL & $\begin{array}{l}\text { GAD-7, PHQ-9, } \\
\text { AIS y SCSQ }\end{array}$ & $\begin{array}{c}\text { El } 35,2 \% \text { de los } \\
\text { sanitarios vs } 23,6 \% \\
\text { de otras poblaciones } \\
\text { presenta elevados } \\
\text { niveles de ansiedad; } \\
\text { y el } 34,4 \% \text { vs } 26,4 \% \\
\text { elevados niveles de } \\
\text { depresión. }\end{array}$ & $\begin{array}{c}\text { Ser mujer. Falta de } \\
\text { apoyo familiar. } \\
\text { Ingreso mensual familiar } \\
\text { bajo. Estilo de vida } \\
\text { sedentario. }\end{array}$ \\
\hline $\begin{array}{l}\text { Guo et al } \\
(2020)\end{array}$ & $\begin{array}{l}\text { Personal sanitario } \\
(\mathrm{n}=11.118) \\
\text { Género femenino: } \\
74,8 \%\end{array}$ & China & ETL & SAS y SDS & $\begin{array}{l}\text { El } 4,98 \% \text { de la muestra } \\
\text { presenta elevados } \\
\text { niveles de ansiedad; y } \\
\text { el } 13,47 \% \text { de depresión. }\end{array}$ & $\begin{array}{l}\text { Ser trabajador sanitario } \\
\text { de primera línea. } \\
\text { Las enfermeras más } \\
\text { afectadas que los } \\
\text { médicos. Falta de } \\
\text { experiencia cuidando } \\
\text { pacientes con } \\
\text { COVID-19 }\end{array}$ \\
\hline $\begin{array}{c}\text { Gupta, } \\
\text { Sahoo, } \\
\text { Mehra y } \\
\text { Grover (2020) }\end{array}$ & $\begin{array}{c}\text { Población general } \\
\text { (n=143) incluyendo } \\
\text { personal sanitario } \\
(n=80) .\end{array}$ & Nepal & ETL & $\begin{array}{c}\text { GAD-7, PHQ-9, } \\
\text { PSS }\end{array}$ & $\begin{array}{l}\text { El } 79 \% \text { de los sanitarios } \\
\text { vs } 88,8 \% \text { de otras } \\
\text { poblaciones presenta } \\
\text { elevados niveles de } \\
\text { estrés; el } 35,4 \% \text { vs } \\
17,5 \% \text { elevados niveles } \\
\text { de ansiedad; y el } 34,3 \% \\
\text { vs } 18,8 \% \text { elevados } \\
\text { niveles de depresión. }\end{array}$ & Ninguno. \\
\hline $\begin{array}{c}\text { Huang y } \\
\text { Zhao (2020) }\end{array}$ & $\begin{array}{c}\text { Población general } \\
\text { ( } \mathrm{n}=7.237) \text { incluyendo } \\
\text { personal sanitario } \\
(\mathrm{n}=2.250) . \\
\text { Edad media: } \\
35,3 \pm 5,6 \\
\text { Género femenino: } \\
54,6 \%\end{array}$ & China & ETL & $\begin{array}{c}\text { CES-D, GAD-7 y } \\
\text { PSQI }\end{array}$ & $\begin{array}{c}\text { El } 23,6 \% \text { de los } \\
\text { sanitarios vs } 18,2 \% \\
\text { de otras poblaciones } \\
\text { presenta alteraciones } \\
\text { del sueño; el } 35,6 \% \text { vs } \\
35,1 \% \text { elevados niveles } \\
\text { de ansiedad y el } 19,8 \% \\
\text { vs } 20,1 \% \text { de depresión. }\end{array}$ & $\begin{array}{l}\text { Obsesión con la } \\
\text { pandemia. }\end{array}$ \\
\hline $\begin{array}{l}\text { Jiang, Nan, } \\
\text { Ly y Yang } \\
\text { (2020) }\end{array}$ & $\begin{array}{c}\text { Población general } \\
\text { ( } \mathrm{n}=6.049 \text { ) incluyendo } \\
\text { personal sanitario } \\
(\mathrm{n}=1.754) . \\
\text { Rango de edad: } 17 \\
\text { a } 73 \\
\text { Género femenino: } \\
74,8 \%\end{array}$ & China & ETL & PCL-C & $\begin{array}{l}\text { El personal sanitario } \\
\text { presenta puntuaciones } \\
\text { de Estrés Postraumático } \\
\text { (moderado a grave) } \\
\text { algo superior al } 19,1 \% \text {. }\end{array}$ & $\begin{array}{l}\text { Ser trabajador de } \\
\text { primera línea. } \\
\text { Trabajar en zonas de } \\
\text { riesgo. Preocupación } \\
\text { por transmitir la } \\
\text { enfermedad a su } \\
\text { familia, particularmente } \\
\text { si tienen hijos. Falta de } \\
\text { apoyo familiar. Tener } \\
\text { familiares confirmados } \\
\text { o con sospecha de } \\
\text { COVID-19 }\end{array}$ \\
\hline $\begin{array}{l}\text { Jungmann } \\
\text { y Witthöft } \\
\text { (2020) }\end{array}$ & $\begin{array}{c}\text { Población general } \\
(\mathrm{n}=1.615) \text { incluyendo } \\
\text { personal sanitario } \\
(\mathrm{n}=161) \\
\text { Edad media: } \\
33,36 \pm 13,2 \\
\text { Género femenino: } \\
79,8 \%\end{array}$ & $\begin{array}{l}\text { Alema- } \\
\text { nia }\end{array}$ & $\begin{array}{l}\text { Encuesta } \\
\text { Iongitudinal } \\
\text { en línea a } 3 \\
\text { puntos: dic- } \\
\text { 19, ene-20 } \\
\text { y mar- } 20 .\end{array}$ & $\begin{array}{c}\text { COVID-19-PTSD } \\
\text { y SHAI }\end{array}$ & $\begin{array}{l}\text { Registran niveles altos } \\
\text { de ansiedad en un } \\
8,3 \%, 19 \% \text { y } 47,18 \% \\
\text { respectivamente. } \\
\text { El personal sanitario } \\
\text { presenta valores de } \\
\text { ansiedad más bajos } \\
\text { que otras poblaciones. }\end{array}$ & $\begin{array}{l}\text { Tener antecedentes de } \\
\text { trastornos mentales. }\end{array}$ \\
\hline $\begin{array}{l}\text { Kang et al. } \\
\text { (2020) }\end{array}$ & $\begin{array}{c}\text { Personal } \\
\text { sanitario=994 } \\
\text { Género femenino: } \\
85,51 \%\end{array}$ & China & ETL & $\begin{array}{c}\text { GAD-7, IES-R, ISI y } \\
\text { PHQ-9 }\end{array}$ & $\begin{array}{l}\text { El } 64 \% \text { de la muestra } \\
\text { presenta elevados } \\
\text { niveles de estrés. }\end{array}$ & $\begin{array}{c}\text { Ser trabajador de } \\
\text { primera línea. } \\
\text { Trabajar en zonas de } \\
\text { riesgo. }\end{array}$ \\
\hline $\begin{array}{l}\text { Lai et al. } \\
\text { (2020) }\end{array}$ & $\begin{array}{l}\text { Personal sanitario } \\
(n=1.257) \\
\text { Género femenino: } \\
76,69 \%\end{array}$ & China & ETL & $\begin{array}{c}\text { GAD-7, IES-R, ISI y } \\
\text { PHQ-9 }\end{array}$ & $\begin{array}{l}\text { El } 34 \% \text { de la muestra } \\
\text { presenta alteraciones } \\
\text { del sueño; el } 71,5 \% \\
\text { presenta elevados } \\
\text { niveles de estrés; el } \\
44,6 \% \text { de ansiedad; y el } \\
50,4 \% \text { de depresión. }\end{array}$ & $\begin{array}{l}\text { Ser Mujer. Enfermeras } \\
\text { más que médicos. }\end{array}$ \\
\hline
\end{tabular}


Tabla 1 (Continuación)

Impacto psicológico y factores de riesgo del COVID-19 en personal sanitario

\begin{tabular}{|c|c|c|c|c|c|c|}
\hline $\begin{array}{l}\text { Li et al. } \\
(2020)\end{array}$ & $\begin{array}{c}\text { Personal de } \\
\text { enfermería de primera } \\
(n=234) \text { y de segunda } \\
\text { línea }(n=292) \text { y otras } \\
\text { poblaciones ( } n=214) \\
\text { Rango de edad: } 22 \\
\text { a } 39 \\
\text { Género femenino: } \\
78,11 \%\end{array}$ & China & ETL & VCQ & $\begin{array}{l}\text { Registran traumatización } \\
\text { vicarial en población } \\
\text { general y personal de } \\
\text { enfermería de segunda } \\
\text { línea }(75,5 \%) \text { mayores } \\
\text { que en personal de } \\
\text { enfermería de primera } \\
\text { línea }(64 \%)\end{array}$ & Ninguno \\
\hline $\begin{array}{l}\text { Liu et al } \\
\text { (2020a) }\end{array}$ & $\begin{array}{l}\text { Personal sanitario } \\
\quad(n=1.563)\end{array}$ & China & ETL & $\begin{array}{c}\text { GAD-7, IES-R, ISI y } \\
\text { PHQ-9 }\end{array}$ & $\begin{array}{l}\text { El } 36,1 \% \text { de la muestra } \\
\text { presenta alteraciones } \\
\text { del sueño; el } 73,4 \% \\
\text { elevados niveles de } \\
\text { estrés; el } 44,7 \% \text { de } \\
\text { ansiedad; y el } 50,7 \% \text { de } \\
\text { depresión. }\end{array}$ & Ninguno \\
\hline $\begin{array}{l}\text { Liu et al } \\
\text { (2020b) }\end{array}$ & $\begin{array}{c}\text { Población general } \\
\text { ( } \mathrm{n}=285 \text { ) incluyendo } \\
\text { personal sanitario } \\
(\mathrm{n}=12) . \\
\text { Género femenino: } \\
54,4 \%\end{array}$ & China & ETL & PSQI y PCL-5 & $\begin{array}{l}\text { Prevalencia de estrés } \\
\text { muy inferiores (aprox. } \\
50 \% \text { ) en personal } \\
\text { sanitario respecto a } \\
\text { positivos por COVID-19 } \\
\text { y personas en contacto } \\
\text { con infectados; y } \\
\text { significativamente } \\
\text { superiores que } \\
\text { población general. }\end{array}$ & $\begin{array}{c}\text { Ser mujer. Mala calidad } \\
\text { del sueño y descanso } \\
\text { inadecuado }\end{array}$ \\
\hline $\begin{array}{l}\text { Liu et al. } \\
\text { (2020c) }\end{array}$ & $\begin{array}{c}\text { Personal sanitario } \\
\text { ( } n=4.679) \\
\text { Edad media: } \\
35,9 \pm 9,0 \\
\text { Género femenino: } \\
\text { 82,26\% }\end{array}$ & China & ETL & $\begin{array}{c}\text { SAS, SDS y SRQ- } \\
20\end{array}$ & $\begin{array}{c}\text { El 15,9\% de la muestra } \\
\text { presenta elevados } \\
\text { niveles de estrés; el 16\% } \\
\text { de ansiedad y el } 34,6 \% \\
\text { de depresión. }\end{array}$ & $\begin{array}{c}\text { Ser Mujer. Falta } \\
\text { de apoyo familiar. } \\
\text { Enfermeras más que } \\
\text { médicos. } \\
\text { Falta de experiencia } \\
\text { cuidando pacientes } \\
\text { con COVID-19. }\end{array}$ \\
\hline $\begin{array}{l}\text { Lu, Wang, Lin } \\
\text { y Li (2020) }\end{array}$ & $\begin{array}{l}\text { Personal sanitario } \\
\quad(n=2.299) \\
\text { Género femenino: } \\
\quad 77,91 \%\end{array}$ & China & ETL & HAMA y HAMD & $\begin{array}{l}\text { El } 25,5 \% \text { de la muestra } \\
\text { presenta elevados } \\
\text { niveles de ansiedad y el } \\
12,1 \% \text { de depresión. }\end{array}$ & Ninguno. \\
\hline $\begin{array}{l}\text { Mertens, } \\
\text { Gerritsen, } \\
\text { Duijndam, } \\
\text { Salemink y } \\
\text { Engelhard } \\
\text { (2020) }\end{array}$ & $\begin{array}{c}\text { Población general } \\
\text { ( } \mathrm{n}=439 \text { ) incluyendo } \\
\text { personal sanitario } \\
(\mathrm{n}=48) \text {. } \\
\text { Rango de edad: } 16 \\
\text { a } 80 \\
\text { Género femenino: } \\
69,9 \%\end{array}$ & $\begin{array}{l}\text { Países } \\
\text { Bajos }\end{array}$ & ETL & $\begin{array}{l}\text { FCQ-8, IUS-12, } \\
\text { PSWQ y SHAI }\end{array}$ & $\begin{array}{c}\text { Ser personal sanitario } \\
\text { no es un predictor de } \\
\text { incremento de miedo a } \\
\text { coronavirus. }\end{array}$ & $\begin{array}{l}\text { Falta de información. } \\
\text { Tener antecedentes de } \\
\text { trastornos mentales. }\end{array}$ \\
\hline Ni et al (2020) & $\begin{array}{c}\text { Población general } \\
\text { ( } \mathrm{n}=1.791 \text { ) incluyendo } \\
\text { personal sanitario } \\
\text { ( } \mathrm{n}=214) \text {, un } 25 \% \text { de } \\
\text { primera línea. } \\
\text { Rango de edad: } 18 \\
\text { a } 60 \\
\text { Género femenino: } \\
68,8 \%\end{array}$ & China & ETL & $\begin{array}{l}\text { GAD-2 y } \\
\text { PHQ-2 }\end{array}$ & $\begin{array}{c}\text { El } 22 \% \text { de los sanitarios } \\
\text { vs } 23,84 \% \text { de la } \\
\text { población general } \\
\text { presenta elevados } \\
\text { niveles de ansiedad y } \\
\text { el } 19,2 \% \text { vs } 19,21 \% \text { de } \\
\text { depresión. }\end{array}$ & $\begin{array}{c}\text { Ser trabajador } \\
\text { de primera línea, } \\
\text { incrementándose si } \\
\text { trabajan en zonas de } \\
\text { riesgo. Obsesión con la } \\
\text { pandemia }\end{array}$ \\
\hline $\begin{array}{l}\text { Odriozola- } \\
\text { González, } \\
\text { Planchuelo- } \\
\text { Gómez, } \\
\text { Irurtia y de } \\
\text { Luis-García } \\
\text { (2020) }\end{array}$ & $\begin{array}{c}\text { Población general } \\
\text { ( } \mathrm{n}=3.550 \text { ) incluyendo } \\
\text { personal sanitario } \\
(\mathrm{n}=346) \\
\text { Edad media: } \\
32.1 \pm 14.1 \\
\text { Género femenino: } \\
64,9 \%\end{array}$ & España & ETL & $\begin{array}{l}\text { DASS-21 y } \\
\text { IES-R }\end{array}$ & $\begin{array}{c}\text { El } 37 \% \text { de la muestra } \\
\text { presenta de elevados } \\
\text { a moderados niveles } \\
\text { de estrés; el } 32,4 \% \text { de } \\
\text { ansiedad y el } 44,1 \% \text { de } \\
\text { depresión. No se ven } \\
\text { incrementados por ser } \\
\text { personal sanitario. }\end{array}$ & $\begin{array}{c}\text { Ser mujer. Aislamiento } \\
\text { social. Tener síntomas } \\
\text { compatibles con } \\
\text { COVID-19. Tener } \\
\text { antecedentes de } \\
\text { trastornos mentales. }\end{array}$ \\
\hline
\end{tabular}


Tabla 1 (Continuación)

Impacto psicológico y factores de riesgo del COVID-19 en personal sanitario

\begin{tabular}{|c|c|c|c|c|c|c|}
\hline $\begin{array}{l}\text { Que et al. } \\
\text { (2020) }\end{array}$ & $\begin{array}{c}\text { Personal sanitario } \\
\text { (n=2.285) } \\
\text { Rango de edad: } 17 \\
\text { a } 64 \\
\text { Género femenino: } \\
\text { 69,06\% }\end{array}$ & China & ETL & $\begin{array}{l}\text { GAD-7, ISI y } \\
\text { PHQ-9 }\end{array}$ & $\begin{array}{l}\text { El 6,78\% de la muestra } \\
\text { presenta alteraciones } \\
\text { del sueño; el 18,12\% } \\
\text { elevados niveles de } \\
\text { estrés; el } 11,6 \% \text { de } \\
\text { ansiedad y el } 12,82 \% \\
\text { de depresión. }\end{array}$ & Ninguno \\
\hline $\begin{array}{l}\text { Romero et al. } \\
\text { (2020) }\end{array}$ & $\begin{array}{c}\text { Personal sanitario } \\
\text { ( } \mathrm{n}=1.631) \\
\text { Edad media: } \\
45,14 \pm 6,48 \\
\text { Género: No } \\
\text { especificado }\end{array}$ & España & ETL & PASS & $\begin{array}{l}\text { Mayor impacto } \\
\text { psicológico en } \\
\text { personal de medicina } \\
\text { respiratoria, seguido del } \\
\text { de geriatría. El estrés es } \\
\text { mayor en áreas más } \\
\text { afectadas, menores de } \\
60 \text { años y en aquellos } \\
\text { que precisan terapia } \\
\text { psicológica y no la } \\
\text { reciben. }\end{array}$ & $\begin{array}{l}\text { Preocupaciones por su } \\
\text { seguridad personal al } \\
\text { desconocer su estado } \\
\text { de salud y el de sus } \\
\text { compañeros. Tener } \\
\text { familiares confirmados } \\
\text { o con sospecha de } \\
\text { COVID-19. }\end{array}$ \\
\hline $\begin{array}{l}\text { Santamaría, } \\
\text { Etxebarria, } \\
\text { Rodriguez, } \\
\text { Albondiga- } \\
\text { Mayor y } \\
\text { Gorrochategui } \\
\text { (2020). }\end{array}$ & $\begin{array}{c}\text { Personal sanitario } \\
(n=421) \\
\text { Rango de edad: } 18 \\
\text { a } 74 \\
\text { Género femenino: } \\
80,3 \%\end{array}$ & España & ETL & DASS-21 y ISI & $\begin{array}{c}\text { El } 28,9 \% \text { de la muestra } \\
\text { presenta alteraciones } \\
\text { del sueño; el } 46,7 \% \\
\text { elevados niveles de } \\
\text { estrés; el } 37 \% \text { de } \\
\text { ansiedad y el } 27,2 \% \text { de } \\
\text { depresión. }\end{array}$ & Ser mujer. \\
\hline $\begin{array}{l}\text { Shacham et } \\
\text { al. (2020) }\end{array}$ & $\begin{array}{c}\text { Personal sanitario } \\
(\mathrm{n}=338) \\
\text { Edad media: } \\
46.4 \pm 11.2 \\
\text { Género femenino: } \\
58,6 \%\end{array}$ & Israel & ETL & DS-S, GSES y K-6 & $\begin{array}{l}\text { El } 11,5 \% \text { de la muestra } \\
\text { presenta elevados } \\
\text { niveles de estrés. }\end{array}$ & Ninguno. \\
\hline $\begin{array}{l}\text { Shechter et al. } \\
\text { (2020) }\end{array}$ & $\begin{array}{l}\text { Personal sanitario } \\
\quad(n=657) \\
\text { Género femenino: } \\
70,9 \%\end{array}$ & EEUU & ETL & $\begin{array}{l}\text { GAD-2, PHQ-2, } \\
\text { PSQl y PC-PTSD }\end{array}$ & $\begin{array}{c}\text { El } 71 \% \text { de la muestra } \\
\text { presenta alteraciones } \\
\text { del sueño; el } 57 \% \\
\text { elevados niveles de } \\
\text { estrés; el } 33 \% \text { de } \\
\text { ansiedad y el } 48 \% \text { de } \\
\text { depresión. }\end{array}$ & $\begin{array}{l}\text { Enfermeras más } \\
\text { que médicos. } \\
\text { Preocupaciones por } \\
\text { su seguridad personal } \\
\text { al desconocer su } \\
\text { estado de salud y el } \\
\text { de sus compañeros. } \\
\text { No tener equipos de } \\
\text { protección adecuados. } \\
\text { Preocupación por } \\
\text { transmitir la enfermedad } \\
\text { a su familia. }\end{array}$ \\
\hline $\begin{array}{l}\text { Shen, Zou, } \\
\text { Zhong, Yan y } \\
\text { Li (2020) }\end{array}$ & $\begin{array}{c}\text { Personal de } \\
\text { enfermería }(n=85) \text { de } \\
\text { primera línea) }\end{array}$ & China & $\begin{array}{c}\text { Entrevista } \\
\text { regular en } \\
\text { línea y cara } \\
\text { a cara. }\end{array}$ & $\begin{array}{l}\text { Cuestionario no } \\
\text { especificado. }\end{array}$ & $\begin{array}{c}\text { El } 45 \% \text { de la muestra } \\
\text { presenta alteraciones } \\
\text { del sueño y el } 59 \% \\
\text { elevados niveles de } \\
\text { estrés. }\end{array}$ & Ninguno. \\
\hline $\begin{array}{l}\text { Sun et al. } \\
(2020 a)\end{array}$ & $\begin{array}{c}\text { Población general } \\
(\mathrm{n}=2.091) \text { incluyendo } \\
\text { personal sanitario } \\
(\mathrm{n}=320) \\
\text { Género femenino: } \\
60,8 \%\end{array}$ & China & ETL & PCL-5 & $\begin{array}{c}\text { Registran niveles de } \\
\text { Estrés Postraumático } \\
\text { del } 4,4 \% \text { en personal } \\
\text { sanitario vs } 5,2 \% \text { en } \\
\text { población general de } \\
\text { bajo riesgo y } 18,4 \% \text { en } \\
\text { población general de } \\
\text { alto riesgo. }\end{array}$ & $\begin{array}{l}\text { Ser mujer. Ser trabajador } \\
\text { de primera línea. } \\
\text { Trabajar en zonas de } \\
\text { riesgo. Mala calidad } \\
\text { del sueño y descanso } \\
\text { inadecuado. }\end{array}$ \\
\hline $\begin{array}{l}\text { Sun et al. } \\
\text { (2020b) }\end{array}$ & $\begin{array}{c}\text { Personal de } \\
\text { enfermería }(n=20) \text { de } \\
\text { primera línea. } \\
\text { Edad media: } \\
30,6 \pm 6,12 \\
\text { Género femenino: } \\
75 \%\end{array}$ & China & $\begin{array}{l}\text { Entrevista } \\
\text { cara a } \\
\text { cara o por } \\
\text { teléfono. }\end{array}$ & $\begin{array}{l}\text { Cuestionario } \\
\text { propio. }\end{array}$ & $\begin{array}{l}\text { El } 75 \% \text { de la muestra } \\
\text { presenta elevados } \\
\text { niveles de estrés y el } \\
50 \% \text { de ansiedad. }\end{array}$ & $\begin{array}{c}\text { Falta de experiencia } \\
\text { cuidando pacientes } \\
\text { con COVID-19. }\end{array}$ \\
\hline
\end{tabular}


Tabla 1 (Continuación)

Impacto psicológico y factores de riesgo del COVID-19 en personal sanitario

\begin{tabular}{|c|c|c|c|c|c|c|}
\hline $\begin{array}{l}\text { Tan et al. } \\
\text { (2020) }\end{array}$ & $\begin{array}{c}\text { Personal sanitario } \\
(n=470) \\
\text { Rango de edad: } 21 \\
\text { a } 36 \\
\text { Género femenino: } \\
68,3 \%\end{array}$ & Singapur & ETL & $\begin{array}{l}\text { DASS-21 y } \\
\text { IES-R }\end{array}$ & $\begin{array}{c}\text { El 7,7\% de la muestra } \\
\text { presenta estrés } \\
\text { postraumático; el 6,6\% } \\
\text { elevados niveles de } \\
\text { estrés; el 14,5\% de } \\
\text { ansiedad y el } 8,9 \% \text { de } \\
\text { depresión. }\end{array}$ & $\begin{array}{l}\text { Ser trabajador sanitario } \\
\text { de segunda línea } \\
\text { (celadores, técnicos, } \\
\text { administrativos, } \\
\text { limpieza, etc.) más que } \\
\text { enfermeras y médicos. }\end{array}$ \\
\hline $\begin{array}{l}\text { Varshney, } \\
\text { Parel, Raizada } \\
\text { y Sarin (2020) }\end{array}$ & $\begin{array}{c}\text { Población general } \\
\text { (n=653) incluyendo } \\
\text { personal sanitario } \\
\text { ( } \mathrm{n}=145 \text { ) } \\
\text { Rango de edad: } 18 \\
\text { a } 82 \\
\text { Género femenino: } \\
23,6\end{array}$ & India & ETL & IES-R & $\begin{array}{c}\text { El 33,3\% de la muestra } \\
\text { presenta elevados } \\
\text { niveles de estrés, } \\
\text { independientemente } \\
\text { de la muestra. }\end{array}$ & Ninguno. \\
\hline $\begin{array}{l}\text { Wang et al. } \\
\text { (2020a) }\end{array}$ & $\begin{array}{c}\text { Personal médico } \\
(n=112) \text { de primera } \\
\text { línea. }\end{array}$ & China & $\begin{array}{l}\text { Entrevista } \\
\text { transversal } \\
\text { cara a } \\
\text { cara. }\end{array}$ & SCL-90-R & $\begin{array}{c}\text { Hay observan } \\
\text { diferencia significativa } \\
\text { en la prevalencia de } \\
\text { ansiedad, somatización, } \\
\text { obsesión, miedo } \\
\text { y psicosis entre el } \\
\text { personal médico y la } \\
\text { población general. }\end{array}$ & $\begin{array}{l}\text { Ser mujer. Ingreso } \\
\text { mensual familiar bajo. }\end{array}$ \\
\hline $\begin{array}{l}\text { Wang et al. } \\
\text { (2020b) }\end{array}$ & $\begin{array}{c}\text { Personal sanitario } \\
(\mathrm{n}=123) \\
\text { Edad media: } \\
33,75 \pm 8,41 \\
\text { Género femenino: } \\
82,11 \%\end{array}$ & China & ETL & PSQI, SAS y SDS & $\begin{array}{c}\text { El } 38 \% \text { de la muestra } \\
\text { presenta alteraciones } \\
\text { del sueño; el } 7 \% \\
\text { elevados niveles de } \\
\text { ansiedad y el } 25 \% \text { de } \\
\text { depresión. }\end{array}$ & Ninguno. \\
\hline $\begin{array}{l}\text { Wu et al. } \\
\text { (2020) }\end{array}$ & $\begin{array}{c}\text { Personal sanitario } \\
(n=2.110) \text { de primera } \\
\text { línea y estudiantes } \\
(n=2.158) \\
\text { Género femenino: } \\
68,9 \%\end{array}$ & China & ETL & PSQ-9 & $\begin{array}{l}\text { El personal médico } \\
\text { del área de Wuhan } \\
\text { presentó mayor } \\
\text { prevalencia de } \\
\text { alteraciones del sueño } \\
\text { y factores de estrés } \\
\text { que otro personal } \\
\text { médico, y éstos más } \\
\text { altos que los estudiantes } \\
\text { universitarios. }\end{array}$ & Ninguno. \\
\hline $\begin{array}{l}\text { Wu y Wei } \\
\text { (2020) }\end{array}$ & $\begin{array}{c}\text { Personal sanitario } \\
(n=120) \\
\text { Rango de edad: } 25 \\
\text { a } 59 \\
\text { Género femenino: } \\
74,17 \%\end{array}$ & China & $\begin{array}{l}\text { Entrevista } \\
\text { cualitativa } \\
\text { cara a } \\
\text { cara. }\end{array}$ & $\begin{array}{l}\text { PSQI, PCL-C, } \\
\text { SCL-90-R y SDS }\end{array}$ & $\begin{array}{c}\text { El } 26,67 \% \text { de la muestra } \\
\text { presenta alteraciones } \\
\text { del sueño. }\end{array}$ & $\begin{array}{l}\text { Ser trabajador de } \\
\text { primera línea. } \\
\text { Trabajar en zonas de } \\
\text { riesgo. }\end{array}$ \\
\hline $\begin{array}{l}\text { Xu, Xu, Wang } \\
\text { y Wang (2020) }\end{array}$ & $\begin{array}{c}\text { Personal sanitario } \\
\text { ( } \mathrm{n}=120) \text { durante el } \\
\text { pico de COVID-19 } \\
\text { y personal sanitario } \\
(\mathrm{n}=60 \text { ) fuera del pico. } \\
\text { Edad media: } \\
36.7 \pm 9.7 \text { vs } 35,8 \pm \\
7,06 \\
\text { Género femenino: } \\
63,3 \% \\
\text { vs } 53,30 \%\end{array}$ & China & $\begin{array}{l}\text { Encuesta } \\
\text { en línea a } 2 \\
\text { tiempos. }\end{array}$ & ISI, SAS y SDS & $\begin{array}{c}\text { El } 46,7 \% \text { de la muestra } \\
\text { en periodo de pico } \\
\text { vs } 10 \% \text { fuera de pico } \\
\text { presenta elevados } \\
\text { niveles de ansiedad } \\
\text { y el } 40 \% \text { vs } 10 \% \text { de } \\
\text { depresión. }\end{array}$ & Ninguno. \\
\hline $\begin{array}{l}\text { Yuang et al. } \\
\text { (2020) }\end{array}$ & $\begin{array}{c}\text { Población general } \\
\text { ( } \mathrm{n}=939) \text { incluyendo } \\
\text { personal sanitario } \\
(\mathrm{n}=249) \\
\text { Género femenino: } \\
62 \%\end{array}$ & China & ETL & PSQI y SRQ & $\begin{array}{l}\text { El 36,43\% de la muestra } \\
\text { presenta alteraciones } \\
\text { del sueño; el } 37,78 \% \\
\text { elevados niveles de } \\
\text { estrés y el } 37,78 \% \\
\text { de somatización. La } \\
\text { ansiedad es superior a } \\
\text { la media en personal } \\
\text { sanitario más expuesto } \\
\text { al CoviD-19, pero no la } \\
\text { calidad del sueño. }\end{array}$ & $\begin{array}{c}\text { Ser trabajador } \\
\text { de primera línea, } \\
\text { incrementándose si } \\
\text { trabajan en zonas de } \\
\text { riesgo. }\end{array}$ \\
\hline
\end{tabular}


Tabla 1 (Continuación)

Impacto psicológico y factores de riesgo del COVID-19 en personal sanitario

\begin{tabular}{|c|c|c|c|c|c|c|}
\hline $\begin{array}{l}\text { Zhang et al } \\
\text { (2020a) }\end{array}$ & $\begin{array}{l}\text { Personal sanitario } \\
\quad(n=1.563) \\
\text { Género femenino: } \\
82,72 \%\end{array}$ & China & ETL & ISI & $\begin{array}{c}\text { El } 36,1 \% \text { de la muestra } \\
\text { presenta alteraciones } \\
\text { del sueño. }\end{array}$ & $\begin{array}{l}\text { Falta de formación } \\
\text { para afrontar el } \\
\text { impacto psicológico. } \\
\text { Aislamiento social. }\end{array}$ \\
\hline $\begin{array}{l}\text { Zhang et al } \\
\text { (2020b) }\end{array}$ & $\begin{array}{c}\text { Población general } \\
\text { ( } \mathrm{n}=2.182 \text { ) incluyendo } \\
\text { personal sanitario } \\
(\mathrm{n}=927) \\
\text { Rango de edad: } 18 \\
\text { a } 60 \\
\text { Género femenino: } \\
64,2 \%\end{array}$ & China & ETL & $\begin{array}{c}\text { ISI, PHQ-2 y } 4 \text { y } \\
\text { SCL-90-R }\end{array}$ & $\begin{array}{l}\text { El } 38,4 \% \text { del personal } \\
\text { sanitario vs } 30.5 \% \text { en } \\
\text { otras poblaciones } \\
\text { presenta insomnio; el } \\
13 \% \text { vs } 8,5 \% \text { elevados } \\
\text { niveles de ansiedad; } \\
\text { el } 12,2 \% \text { vs } 9,5 \% \text { de } \\
\text { depresión; el 1,6\% vs } \\
0,4 \% \text { de somatización; } \\
\text { y el } 5,3 \% \text { vs } 2,2 \% \text {. } \\
\text { de Síntomas obsesivo- } \\
\text { convulsivos. }\end{array}$ & $\begin{array}{c}\text { Ser mujer. Ser } \\
\text { trabajador sanitario de } \\
\text { segunda línea más que } \\
\text { personal médico. }\end{array}$ \\
\hline $\begin{array}{l}\text { Zhu et al. } \\
\text { (2020a) }\end{array}$ & $\begin{array}{c}\text { Personal sanitario } \\
(\mathrm{n}=5.060) \\
\text { Rango de edad: } 19 \\
\text { a } 49 \\
\text { Género femenino: } \\
\quad 85 \%\end{array}$ & China & ETL & $\begin{array}{c}\text { GSES, IES-R Y } \\
\text { PSQI }\end{array}$ & $\begin{array}{c}\text { El } 29,8 \% \text { de la muestra } \\
\text { presenta elevados } \\
\text { niveles de estrés; el } \\
24,1 \% \text { de ansiedad y el } \\
13,5 \% \text { de depresión. }\end{array}$ & $\begin{array}{l}\text { Ser mujer. Tener } \\
\text { antecedentes de } \\
\text { trastornos mentales. } \\
\text { Tener familiares } \\
\text { confirmados O con } \\
\text { sospecha de COVID-19 }\end{array}$ \\
\hline $\begin{array}{l}\text { Zhu et al. } \\
\text { (2020b) }\end{array}$ & $\begin{array}{c}\text { Personal de } \\
\text { enfermería }(n=86) \\
\text { y personal médico } \\
(n=79) \\
\text { Edad } \\
\text { media: } 34,16 \pm 8,06 \\
\text { Género femenino: } \\
83 \%\end{array}$ & China & ETL & SAS y SDS & $\begin{array}{c}\text { El 1 1,4\% personal } \\
\text { médico vs } 27,9 \% \\
\text { personal enfermería } \\
\text { presenta elevados } \\
\text { niveles de ansiedad } \\
\text { y el } 45,6 \% \text { vs } 43 \% \text { de } \\
\text { depresión. }\end{array}$ & $\begin{array}{l}\text { Ser mujer. Tener } \\
\text { antecedentes de } \\
\text { trastornos mentales. }\end{array}$ \\
\hline \multicolumn{7}{|c|}{$\begin{array}{l}\text { Athens Insomnia Scale (AIS). Beck Ansiedad Inventory (BAI). Beck Depresión Inventory (BDI). Connor-Davidson resilience scale (CD-RISC). } \\
\text { Center for Epidemiology Scale for Depresión (CES-D). COVID-19 Posttraumatic Stress Disorder (COVID-19-PTSD). Depresión, Ansiedad } \\
\text { and Stress Scale (DASS-21). Demands Scale-Short Version (DS-S). Fear of the Coronavirus Questionnaire-8 (FCQ-8). Generalized } \\
\text { Ansiedad Disorder (GAD-2) and (GAD-7). General Self-Efficacy Scale (GSES). Hamilton Ansiedad Scale (HAMA). Hamilton Depresión } \\
\text { Scale (HAMD). Impact of Event Scale-Revised (IES-R). Insomnia Severity Index (ISI). Intolerance of Uncertainty Scale (IUS-12). Kessler } 6 \\
\text { Estrés Scale (K-6). Maslach Burn-out Inventory (MBI). No especificado (NS). Cuestionario propio (OQ). Perinatal Ansiedad Screening } \\
\text { Scale (PASS). Primary Care PTSD screen (PC-PTSD). Posttraumatic Stress Disorder Checklist for DSM-5 (PCL-5). Posttraumatic Stress } \\
\text { Disorder Checklist- Civilian Version (PCL-C). Patient Health Questionnaire (PHQ-2), (PHQ-4) y (PHQ-9). Pittsburgh Sleep Quality Index } \\
\text { (PSQI). Perceived Stress Scale (PSS). Penn State Worry Questionnaire (PSWQ). Self-Rating Ansiedad Scale (SAS). Self-Rating Depresión } \\
\text { Scale (SDS). Symptom Check List-revised (SCL-90-R). Simplified Coping Style Questionnaire (SCSQ). Short Health Ansiedad Inventory } \\
\text { (SHAI). Stress Response Questionnaire (SRQ). Social Support Rate Scale (SSRS). Vicarious traumatization questionnaire (VCQ). }\end{array}$} \\
\hline
\end{tabular}

En relación a los factores de riesgo, las mayores prevalencias dependen de factores relacionados con situaciones de más riesgo y pertenencia a grupos poblacionales más susceptibles, pudiendo identificar los siguientes:

1. Ser trabajador sanitario de segunda línea (celadores, técnicos, administrativos, limpieza, etc.) más que personal de enfermería (Tan et al., 2020; Zhang et al., 2020b); y éstos más que personal médico (Guo et al., 2020; Lai et al., 2020; Liv et al., 2020c; Shechter et al. 2020).
2. Ser trabajador de primera línea, incrementándose en zonas de riesgo (Jiang et al (2020); Kang et al., 2020; Ni et al., 2020; Sun et al., 2020a; Wu y Wei, 2020; Yuang et al., 2020).

3. Ser mujer (Du et al., 2020; Fu et al., 2020; Lai et al., 2020; Liu et al., 2020bc; Odriozola-González et al., 2020; Santamaría et al., 2020; Sun et al., 2020a; Wang et al., 2020a; Zhang et al., 2020b; Zhu et al., 2020ab). 
4. Falta de información (Mertens et al., 2020), formación (Abdessater et al., 2020) y experiencia cuidando pacientes con COVID- 19 (Cai et al., 2020b; Chen et al.2020b;, Guo et al., 2020; Liu et al., 2020c; Sun et al., 2020b).

5. Preocupaciones por su seguridad personal al desconocer su estado de salud y el de sus compañeros (Romero et al., 2020; Shechter et al., 2020), no tener directrices claras para trabajar (Abdessater et al., 2020) ni equipos de protección adecuados (Abdessater et al., 2020; Cai et al., 2020a; Shechter et al., 2020).

6. Preocupación por los pacientes y sensación de incapacidad para cuidarlos adecuadamente (Cai et al., 2020a; Du et al., 2020).

7. Preocupación por transmitir la enfermedad a su familia (Cai et al., 2020a; Shechter et al., 2020) particularmente si tienen hijos (Jiang et al., 2020).

8. Agotamiento por exceso de trabajo (Cai et al., 2020a), mala calidad del sueño y descanso inadecuado (Du et al., 2020; Liu et al., 2020b; Sun et al., 2020a).

9. Falta de formación para afrontar el impacto psicológico (Du et al., 2020; Zhang et al, 2020b).

10. Aislamiento social (Odriozola-González et al., 2020; Zhang et al., 2020b) y falta de apoyo familiar (Du et al., 2020; Fu et al., 2020; Jiang et al., 2020; Liu et al., 2020c).

11. Tener antecedentes de trastornos mentales (Jungmann y Witthöft, 2020; Mertens et al., 2020; Odriozola- González et al., 2020; Zhu et al., 2020ab)

12. Tener síntomas compatibles con COVID-19 (Chew et al., 2020; OdriozolaGonzález et al., 2020).

13. Tener familiares confirmados o con sospecha de COVID-19 (Jiang et al., 2020; Romero et al., 2020; Zhu et al., 2020a).

14. Obsesión con la pandemia (Huang y Zhao, 2020a; Ni et al., 2020).

15. Ingreso mensual familiar bajo (Fu et al., 2020; Wang et al. 2020a).

16. Estilo de vida sedentario (Fu et al., 2020).

Tabla 2

Análisis de Intervenciones psicológicas

\begin{tabular}{|c|c|c|c|c|}
\hline Referencia & Muestra & Origen & Intervención & Resultados \\
\hline Buselli et al. (2020) & $\begin{array}{c}\text { Personal sanitario } \\
\quad(n=106) \\
\text { Edad: } 28 \text { a } 66 \\
\text { Género femenino: } \\
74,53 \%\end{array}$ & Italia & $\begin{array}{l}\text { Programa de intervención } \\
\text { PISOCOVID-19. Utiliza el correo } \\
\text { electrónico para facilitar el } \\
\text { contacto rápido y anónimo. } \\
\text { Hacen el triaje mediante } \\
\text { Ilamada telefónica. Aplican } \\
\text { (en línea o cara a cara) } \\
\text { intervenciones cognitivo- } \\
\text { conductuales adecuadas a } \\
\text { cada caso. }\end{array}$ & No se aportan. \\
\hline Chen et al. (2020) & $\begin{array}{l}\text { Personal sanitario } \\
\quad(n=13)\end{array}$ & China & $\begin{array}{l}\text { Efectúan el diagnóstico y } \\
\text { seguimiento mediante entrevista } \\
\text { cualitativa cara a cara. }\end{array}$ & $\begin{array}{l}\text { Es necesario ayudar a solucionar } \\
\text { sus problemas inmediatos (miedo a } \\
\text { contagiar a su familia, desconocimiento } \\
\text { de la enfermedad, preocupación por } \\
\text { la escasez de equipos de protección, } \\
\text { necesidad de más descanso } \\
\text { sin interrupciones y sentimientos } \\
\text { de incapacidad ante pacientes } \\
\text { críticamente enfermos) para que la } \\
\text { ayuda psicológica sea efectiva. Y dicen } \\
\text { observar mejora tras aplicar medidas al } \\
\text { respecto. }\end{array}$ \\
\hline
\end{tabular}


Tabla 2 (Continuación)

Análisis de Intervenciones psicológicas

\begin{tabular}{|c|c|c|c|c|}
\hline Referencia & Muestra & Origen & Intervención & Resultados \\
\hline Jiang (2020) & $\begin{array}{l}\text { Personal sanitario ( } \mathrm{n} \\
\quad=534 \text { ) } \\
\text { Edad: } 18 \text { a } 40 \\
\text { Género femenino: } \\
\quad 68,73 \%\end{array}$ & China & $\begin{array}{c}\text { Analiza la eficacia percibida } \\
\text { de las medidas hospitalarias } \\
\text { aplicadas para el control del } \\
\text { estrés. }\end{array}$ & $\begin{array}{c}\text { Observaron un impacto positivo tras } \\
\text { aplicar intervenciones psicológicas de } \\
\text { afrontamiento y aumentar la provisión } \\
\text { de instalaciones y equipos en el } \\
\text { hospital. }\end{array}$ \\
\hline $\begin{array}{l}\text { Romero et al. } \\
\text { (2020) }\end{array}$ & $\begin{array}{l}\text { Personal sanitario ( } \mathrm{n} \\
\quad=1631 \text { ) } \\
\text { Edad media: } \\
45,14 \pm 6,48\end{array}$ & España & $\begin{array}{l}\text { Analizan la necesidad auto- } \\
\text { percibida y su impacto en } \\
\text { los programas de atención } \\
\text { psicológica. }\end{array}$ & $\begin{array}{l}\text { Los trabajadores que necesitaron } \\
\text { terapia psicológica y no la recibieron } \\
\text { estaban más estresados que los que no } \\
\text { la necesitaron. }\end{array}$ \\
\hline $\begin{array}{l}\text { Shen, Zou, Zhong, } \\
\text { Yan y Li (2020) }\end{array}$ & $\begin{array}{l}\text { Personal enfermería } \\
\text { de UCl }(n=85)\end{array}$ & China & $\begin{array}{l}\text { Programa de intervención } \\
\text { con medidas tempranas, un } \\
\text { psicólogo por grupo médico, } \\
\text { reuniones periódicas (en línea y } \\
\text { cara a cara) para encontrar las } \\
\text { fuentes de los problemas y dar } \\
\text { soluciones específicas. }\end{array}$ & $\begin{array}{l}\text { No aportan datos cuantitativos } \\
\text { ni cualitativos, pero concluyen } \\
\text { recomendando un programa de } \\
\text { intervención completo. }\end{array}$ \\
\hline Sun et al. (2020b) & $\begin{array}{c}\text { Personal enfermería } \\
\text { de primera línea ( } \mathrm{n} \\
=20 \text { ) } \\
\text { Edad media: } 30,60 \\
\pm 6,12 \\
\text { Género femenino: } \\
75 \%\end{array}$ & China & $\begin{array}{l}\text { Valoran la eficacia de las } \\
\text { técnicas de adaptación } \\
\text { utilizadas por el personal (escribir } \\
\text { diarios y cartas, relajación } \\
\text { respiratoria, atención plena, } \\
\text { meditación musical y expresión } \\
\text { emocional y desahogo). }\end{array}$ & $\begin{array}{l}\text { En la fase previa a formación para } \\
\text { entrar a la sala de presión negativa, la } \\
\text { prevalencia es del } 75 \% \text { para estrés y del } \\
50 \% \text { para ansiedad. Tras una semana la } \\
\text { prevalencia de emociones negativas se } \\
\text { reduce al } 30 \% \text {. }\end{array}$ \\
\hline $\begin{array}{l}\text { Xiao, Zhang, Kong, } \\
\text { Li y Yang (2020) }\end{array}$ & $\begin{array}{l}\text { Personal sanitario ( } \mathrm{n} \\
\quad=180 \text { ) } \\
\text { Edad media: } 32,31 \\
\quad \pm 4,88 \\
\text { Género femenino: } \\
\quad 71,70 \%\end{array}$ & China & $\begin{array}{c}\text { Analizan la importancia del } \\
\text { apoyo social como medida de } \\
\text { apoyo. }\end{array}$ & $\begin{array}{c}\text { El apoyo social aumenta la calidad del } \\
\text { sueño, al reducir la ansiedad y el estrés } \\
\text { y aumentar la autoeficacia. }\end{array}$ \\
\hline
\end{tabular}

\section{- INTERVENCIONES PSICOLÓGICAS}

Se han seleccionado 7 artículos empíricos (ver Tabla 2) sobre intervenciones psicológicas. Estos trabajos reportan programas basados en la solución de los problemas inmediatos del personal sanitario relacionados con su seguridad personal y familiar, la falta de materiales y equipos de protección, el descanso efectivo y la falta de formación para atender a estos pacientes (Chen et al., 2020a) y equipos de intervención psicológica temprana que (a) enseñen técnicas psicológicas adaptativas para enfrentar la carga psicológica originada por el COVID-19, (Du et al., 2020; Zhang et al., 2020b) como escribir diarios y cartas, la relajación respiratoria, actividades de atención plena, meditación musical o expresión emocional y desahogo (Sun et al., 2020b); (b) efectúen triajes en línea (Liv et al., 2020a) o por teléfono (Buselli et al., 2020); (c) desarrollen actividades grupales para reducir el estrés; y (d) den consejo profesional cara a cara (Buselli et al., 2020; Chen et al., 2020a; Sen et al., 2020) o en línea (Liv et al., 2020a; Buselli et al., 2020). Para el tratamiento aplican intervenciones cognitivo-conductuales para 
mejorar habilidades de regulación emocional basadas en la aceptación, enfrentar la ansiedad y el miedo al contagio; restaurar la resiliencia personal frente al estrés (Buselli et al., 2020), aceptar sus limitaciones, aprender a perdonarse a sí mismos y establecer sistemas de apoyo social (Wu et al. (2020).

\section{DISCUSIÓN}

En comparación con la única revisión sistemática hallada sobre el tema (Pappa et at., 2020), en nuestro estudio hemos ampliado la muestra y su origen, y hemos incluido los estudios sobre intervenciones psicológicas. Además, frente a los estudios hallados (Luo et al., 2020; Pappa et al., 2020; Rajkumar, 2020), que se centran en artículos en lengua inglesa, hemos ampliado la búsqueda a artículos en español, inglés, francés, italiano y portugués.

Para el impacto psicológico y los factores de riesgo asociados se ha analizado una muestra total de 48.830 sanitarios procedentes de Alemania, Canadá, China, Estados Unidos, Francia, India, Irán, Israel, Italia, Nepal, Países Bajos, Singapur y España, si bien prevalece la muestra China (artículos: 68,08\%; muestra: 87,22\%). En general, los estudios presentan grandes muestras, pero dado que la mayoría de estudios realizaron un muestreo incidental, apenas se registraron datos de personas mayores de 60 años, con porcentajes superiores de mujeres que, de hombres, salvo en los estudios de Ashtari et al. (2020) y Varshney et al. (2020) donde sucedía a la inversa. En los estudios de Consolo et al. (2020), Gupta et al. (2020), Liu et al. (2020a), Romero et al. (2020), Shen et al. (2020) y Wang et al. (2020a) no se aportaba información sobre el género.

Otra limitación hallada en los estudios es la falta de concreción en cuanto a su labor profesional. Algunos, como Chen et al. (2020ab), Li et al. (2020) y Zhu et al. (2020b), analizan personal médico y/o de enfermería, pero en general solo hablan de personal sanitario. Tan et al. (2020) y Zhang et al. (2020b) aportan datos de otros profesionales, pero incluyen a celadores y técnicos de ambulancia en el mismo grupo que personal de limpieza y administrativos, sin tener en cuenta el diferente contacto que tienen con enfermos o zonas contaminadas.
Se observa, falta de consenso en los cuestionarios a utilizar (reportando el uso de 37 diferentes) y en los puntos de corte; o como ocurre en el estudio de Ashtari et al. (2020) no se establece punto de corte, por lo que los datos solo pueden ser considerados a nivel comparativo entre poblaciones. Además, aunque los más comunes son cuestionarios validados conocidos, algunos han sido especialmente diseñados para evaluar el miedo (Mertens et al., 2020), el estrés (Cai et al., 2020a; Sun et al., 2020b) o el estrés postraumático (Jiang et al., 2020) por COVID-19; o son abreviaturas de cuestionarios validados (Fu et al., 2020; Mertens et al., 2020; $\mathrm{Ni}$ et al., 2020; Shacham et al., 2020; Shechter et al., 2020; Zhang et al., 2020b).

Prevalecen los estudios transversales a corto plazo y ninguno de ellos aporta datos prepandémicos, por lo que los investigadores comparan los resultados con datos de estudios previos. Algo similar ocurre con los longitudinales. A su vez, la necesidad de aislamiento social generado por la pandemia justifica que más del 92\% de los estudios sean mediante entrevista en línea y que en los realizados cara a cara la muestra haya sido muy reducida. A este respecto, aunque el volumen de muestra puede reducir el sesgo, no debemos olvidar que, tal y como indica Luo et al. (2020), los autoinformes presentan riesgo de deseabilidad social, la entrevista en línea tiene un mayor riesgo de falso juicio o sobre juicio y las redes sociales pueden presentar una retroalimentaciones irracionales y engañosas. A su vez, muchas personas no utilizan las redes sociales usadas, entre otros, por Du et al. (2020), Guo et al. (2020) o Kang et al. (2020); y su uso es menor en personas poco formadas, de bajo nivel económico o de mayor edad, lo cual puede explicar el bajo número de datos recopilados en personal sanitario de soporte y de mayores de 60 años.

Tal y como se indica en resultados, los datos de prevalencia muestran resultados muy variables, posiblemente debido a la fase de la pandemia en el momento del estudio y los factores de riesgo asociados a la muestra. También, se observan diferencias entre países orientales y occidentales, con valores mínimos más altos en estos últimos. Ello puede ser debido a que en los países orientales los estudios cubren la evolución 
completa de la pandemia en tanto que en los occidentales solo se cubre la fase de escalada. Así, por ejemplo, en el estudio longitudinal efectuado por Jungmann y Witthöft (2020) en Alemania, obtuvieron datos en personal sanitario que indican prevalencias de ansiedad del 8,3\% en diciembre (pre-pandemia), del 19\% en enero (alerta de pandemia) y del $47,18 \%$ en marzo (escalada de pandemia). Este último dato se aproxima al dado por Consolo et al. (2020), Lai et al., (2020), Liv et al. (2020a) o Xu et al. (2020), para la misma fase de pandemia. El estudio no reporta datos posteriores, donde, teniendo en cuenta los datos aportados por Cai et al. (2020b), Chen et al. (2020b), Guo et al. (2020), Liv et al. (2020c) o Sun et al. (2020b), se podía esperar una disminución de la prevalencia. Por su parte, los estudios comparativos con otras poblaciones muestran una gran variabilidad, con datos de prevalencia en estrés, ansiedad, y depresión que van de superiores a inferiores con respecto a población general; datos que pueden estar relacionados con los factores de riesgo modificables propios de este grupo de población y las intervenciones llevadas a cabo en los servicios sanitarios correspondientes.

Por otro lado, para esta revisión se seleccionaron 7 artículos empíricos (Tabla 2) sobre intervenciones psicológicas; analizando 2.567 profesionales sanitarios de España (63,53\%), China (32,33\%) e Italia (4,13\%). Los trabajos no muestran una línea común de actuación, por lo que no son comparativos. A su vez, son estudios transversales, observacionales y auto-informados, por lo que, ante la falta de datos concretos a medio o largo plazo, no ha sido posible establecer su efectividad. En todo caso, se confirma su demanda y aceptación por los profesionales sanitarios (Chen et al., 2020; Jiang, 2020, Sun et al., 2020b).

En conclusión, a pesar de las limitaciones metodológicas referenciadas, 0 el déficit de información sobre personal sanitario de apoyo (auxiliares de enfermería, técnicos de emergencias, personal de limpieza hospitalaria, etc.), la evidencia hallada permite alcanzar los objetivos planteados en este estudio. Los datos confirman el alto impacto psicológico en estos profesionales, con altas prevalencias de estrés, alteraciones del sueño, ansiedad, depresión y estrés postraumático, que dependen de factores relacionados con situaciones de mayor riesgo y con la pertenencia a grupos poblacionales más susceptibles. También permite hacer una aproximación a las intervenciones llevadas a cabo y, aunque no ha sido posible establecer su eficacia, si se confirma su demanda y aceptación por los profesionales sanitarios. En todo caso, es necesario seguir investigando para obtener datos a largo plazo teniendo en cuenta los diferentes grupos y sus niveles de riesgo.

\section{- Conflicto de intereses.}

Los autores declaran no tener ningún conflicto de intereses.

\section{REFERENCES}

*Abdessater, M., Rouprêt, M., Misrai, V., Pinar, U., Matillon, X., Gondran-Tellier, B., ... \& Pradere, B. (2021). COVID-19 outbreak situation and its psychological impact among surgeons in training in France. World Journal of Urology, 39(3), 971-972. https://dx.doi. org/10.1007/s00345-020-03207-x

Álvarez-Muelas, A., Gómez-Berrocal, C., \& Sierra, JC. (2000). Relación del doble estándar sexual con el funcionamiento sexual y las conductas sexuales de riesgo: Revisión sistemática. [Relationship of sexual double standard with sexual functioning and risk sexual behaviors: A systematic review]. Revista Iberoamericana de Psicología y Salud, 9(2). https://doi.org/10.23923/i. rips.2020.02.038

*Buselli, R., Baldanzi, S., Corsi, M., Chiumiento, M., Del Lupo, E., et al. (2020). Psychological Care of Health Workers during the COVID-19 Outbreak in Italy: Preliminary Report of an Occupational Health Department (AOUP) Responsible for Monitoring Hospital Staff Condition. Sustainability, 12(12), 5039. https://doi.org/10.3390/su 12125039

*Cai, H., Tu, B., Ma, J., Chen, L., Fu, L., et al. (2020a). Psychological impact and coping strategies of frontline medical staff in hunan between january and march 2020 during the outbreak of coronavirus disease 2019 (COVID-19) in Hubei, China. Medical science monitor: international 
medical journal of experimental and clinical research, 26, e924171-1. https://dx.doi. org/10.12659\%2FMSM.924171

Cai, W., Lian, B., Song, X., Hou, T., Deng, G., \& Li, H. (2020b). A cross-sectional study on mental health among health care workers during the outbreak of Corona Virus Disease 2019. Asian Journal of Psychiatry, 51, 102111. https://doi.org/10.1016/i. aip.2020.102111

Cao, J., Wei, J., Zhu, H., Duan, Y., Geng, W., et al. (2020). A study of basic needs and Psychological wellbeing of medical workers in the fever clinic of a tertiary general hospital in Beijing during the COVID-19 outbreak. Psychotherapy and Psychosomatics. Advance online publication. https://dx.doi. org/10.1159/000507453

*Chen, Q., Liang, M., Li, Y., Guo, J., Fei, D., et al. (2020a). Mental health care for medical staff in China during the COVID-19 outbreak. The Lancet Psychiatry, 7(4), e15-e16. https:// doi.org/10.1016/S2215-0366(20)30078-X

Chen, Y., Zhou, H. , Zhou, Y., \& Zhou, F. (2020b). Prevalence of self-reported depression and anxiety among pediatric medical staff members during the COVID-19 outbreak in Guiyang, China. Psychiatry Research. Advance online publication. https://dx.doi. org/10.1016\%2Fi. psychres.2020.113005

Chew, N.W., Lee, G.K., Tan, B.Y., Jing, M., Goh, Y., ... \& Sharma., V. (2020). A multinational, multicentre study on the psychological outcomes and associated physical symptoms amongst healthcare workers during COVID-19 outbreak. Brain, Behavior, and Immunity, 88, 559-565. https://doi. org/10.1016/i.bbi.2020.04.049

${ }^{*}$ Chung, JP., \& Yeung, W. S. (2020). Staff mental health self-assessment during the COVID-19 outbreak. East Asian Archives of Psychiatry, 30(1), 34.

*Consolo, U., Bellini, P., Bencivenni, D., lani, C., \& Checchi, V. (2020). Epidemiological Aspects and Psychological Reactions to COVID-19 of Dental Practitioners in the Northern Italy Districts of Modena and Reggio Emilia. International Journal of
Environmental Research and Public Health, 17(10), 3459. https://doi.org/10.3390/ ijerph 17103459

Du, J., Dong, L., Wang, T., Yuan, C., Fu, R., Zang, L., ... \& Li., X. (2020). Psychological symptoms among frontline healthcare workers during COVID-19 outbreak in Wuhan. General Hospital Psychiatry, 67, 144-145. https://dx.doi.org/10.1016\%2Fi. genhosppsych.2020.03.011

Fariña, F., Arce, R. y Novo, M. (2002). Heurístico de anclaje en las decisiones judiciales [Anchorage in judicial decision making]. Psicothema, 14, 39-46. Recuperado de: http://www.psicothema.com/pdf/684.pdf

*Fu, W., Wang, C., Zou, L., Guo, Y., Lu, Z., et al. (2020). Psychological health, sleep quality, and coping styles to stress facing the COVID-19 in Wuhan, China. Translational psychiatry, 10(1), 1-9. https://dx.doi. org/10.1038\%2Fs41398-020-00913-3

Guo, J., Liao, L., Wang, B., Li, X., Guo, L., et al. (2020). Psychological Effects of COVID-19 on Hospital Staff: A National Cross-Sectional Survey of China Mainland. Advance online publication. https://dx.doi. org/10.2139/ssrn.3550050

Gupta, AK., Sahoo, S., Mehra, A., \& Grover, S. (2020). Psychological impact of 'Lockdown'due to COVID-19 pandemic in Nepal: An Online Survey. Asian Journal of Psychiatry. Advance online publication. https://dx.doi.org/10.1016\%2Fi. aip. 2020.102243

Higgins, JP., Savović, J., Page, MJ., Elbers, RG., \& Sterne, JA. (2019). Assessing risk of bias in a randomized trial. Cochrane Handbook for Systematic Reviews of Interventions, 205-228. https://doi. org/10.1002/9781119536604.ch8

Hoffmann, TC., Glasziou, PP., Boutron, I., Milne, R., Perera, R., et al. (2016). Better reporting of interventions: template for intervention description and replication (TIDieR) checklist and guide. Gesundheitswesen (Bundesverband der Arzte des Offentlichen Gesundheitsdienstes (Germany), 78(3), 175. https://doi.org/10.1055/s-0041-111066 
Huang, Y., \& Zhao, N. (2020a). Generalized anxiety disorder, depressive symptoms and sleep quality during COVID-19 outbreak in China: a web-based cross-sectional survey. Psychiatry Research, 263, 292-300. https:// doi.org/10.1016/i.psychres.2020.112954

*Jiang, Y. (2020). Psychological impact and coping strategies of frontline medical staff in Hunan between January and March 2020 during the outbreak of Coronavirus Disease 2019 (COVID 19) in Hubei, China. Med Sci Monit, 26, e924171. https://doi. org/10.12659/MSM.924171

*Jiang, HJ., Nan, J., Lv, ZY., \& Yang, J. (2020). Psychological impacts of the COVID-19 epidemic on Chinese people: Exposure, post-traumatic stress symptom, and emotion regulation. Asian Pacific Journal of Tropical Medicine, 13(6), 252. https://dx.doi. org/10.4103/1995-7645.281614

Jungmann, S. M., \& Witthöft, M. (2020). Health anxiety, cyberchondria, and coping in the current COVID-19 pandemic: Which factors are related to coronavirus anxiety? Journal of Anxiety Disorders, 73, 102239. https:// doi.org/10.1016/i.janxdis.2020.102239

Kang, L., Ma, S., Chen, M., Yang, J., Wang, Y., Li, R., ... \& Liv, Z. (2020). Impact on mental health and perceptions of psychological care among medical and nursing staff in Wuhan during the 2019 novel coronavirus disease outbreak: A cross-sectional study. Brain, Behavior, and Immunity, 87, 11-17. https:// doi.org/10.1016/i. bbi.2020.03.028

*Lai, J., Ma, S., Wang, Y., Cai, Z., Hu, J., et al. (2020). Factors associated with mental health outcomes among health care workers exposed to coronavirus disease 2019. JAMA network open, 3(3), e203976-e203976. https://doi.org/10.1001/ jamanetworkopen.2020.3976

Li, Z., Ge, J., Yang, M., Feng, J., Qiao, M., Jiang, R., ... \& Yang, C. (2020). Vicarious traumatization in the general public, members, and non-members of medical teams aiding in COVID-19 control. Brain, Behavior, and Immunity, 88, 916-919. https:// doi. org/10.1016/i.bbi.2020.03.007
Liberati, A., Altman, DG., Tetzlaff, J., Mulrow, C., Gøtzsche, PC., et al. (2009). The PRISMA statement for reporting systematic reviews and meta-analyses of studies that evaluate health care interventions: explanation and elaboration. Journal of clinical epidemiology, 62(10), el-e34. https://doi.org/10.1371/ journal.pmed. 1000097

*Liu, S., Yang, L., Zhang, C., Xiang, Y. T., Liu, Z., et al. (2020a). Online mental health services in China during the COVID-19 outbreak. The Lancet Psychiatry, 7(4), e 17-e 18. https://doi. org/10.1016/S2215-0366(20)30077-8

Liu, N., Zhang, F., Wei, C., Jia, Y., Shang, Z., Sun, L ... \& Liu, W. (2020b). Prevalence and predictors of PTSS during COVID-19 outbreak in China hardest-hit areas: Gender differences matter. Psychiatry Research, 287, 112921. https://doi.org/10.1016/i. psychres.2020.112921

Liv, Z., Han, B., Jiang, R., Huang, Y., Ma, C., Wen, J., ... \& Ma, Y. (2020). Mental health status of doctors and nurses during COVID- 19 epidemic in China. Advance online publication. https://dx.doi.org/10.2139/ ssrn.3551329

Lu, W., Wang, H., Lin, Y., \& Li, L. (2020). Psychological status of medical workforce during the COVID-19 pandemic: A crosssectional study. Psychiatry Research, Advance online publication. https://doi.org/10.1016/i. psychres.2020.112936

Luo, M., Guo, L., Yu, M., \& Wang, H. (2020). The psychological and mental impact of coronavirus disease 2019 (COVID-19) on medical staff and general public-a systematic review and meta-analysis. Psychiatry Research, $173190 . \quad$ https://doi.org/10.1016/i. psychres.2020.113190

Mertens, G., Gerritsen, L., Duijndam, S., Salemink, E., \& Engelhard, I.M. (2020). Fear of the coronavirus (COVID-19): Predictors in an online study conducted in March 2020. Journal of Anxiety Disorders, 74, 102258. https://doi. org/10.1016/i.janxdis.2020.102258

Monteiro, A., José Vázquez, M., Seijo, D., \& Arce, R. (2018). ¿̇Son los criterios de realidad válidos para clasificar y discernir entre 
memorias de hechos auto-experimentados y de eventos vistos en vídeo? [Are the reality criteria valid to classify and to discriminate between memories of self-experienced events and memories of video-observed events?] Revista Iberoamericana de Psicología y Salud, 9(2), 149-160. https://doi.org/10.23923/i. rips.2018.02.020

*Ni, MY., Yang, L., Leung, CM., Li, N., Yao, XI., et al. (2020). Mental health, risk factors, and social media use during the COVID-19 epidemic and cordon sanitaire among the community and health professionals in Wuhan, China: Cross-sectional survey. JMIR mental health, 7(5), e 19009. https://dx.doi. org/10.2196\%2F19009

*Odriozola-González, P., Planchuelo-Gómez, Á., Irurtia-Muñiz, M. J., \& de Luis-García, R. (2020). Psychological symptoms of the outbreak of the COVID-19 crisis and confinement in the population of Spain. Recuperado de: https://doi.org/10.31234/ osf.io/ma4fg

Pappa, S., Ntella, V., Giannakas, T., Giannakoulis, V. G., Papoutsi, E., \& Katsaounou, P. (2020). Prevalence of depression, anxiety, and insomnia among healthcare workers during the COVID-19 pandemic: A systematic review and meta-analysis. Brain, Behavior, and Immunity, 88, 901-907. https://doi. org/10.1016/i.bbi.2020.05.026

*Que, J., Le Shi, JD., Liu, J., Zhang, L., Wu, S., et al. (2020). Psychological impact of the COVID-19 pandemic on healthcare workers: a cross-sectional study in China. General Psychiatry, 33(3). https://dx.doi. org/10.1 136\%2Fgpsych-2020-100259

Rajkumar, R. P. (2020). COVID-19 and mental health: A review of the existing literature. Asian Journal of Psychiatry, 52, 102066. https:// doi.org/10.1016/i.aip.2020.102066

Romero, C. S., Delgado, C., Catalá, J., Ferrer, C., Errando, C., Iftimi, A., ... \& Otero, M. (2020). COVID-19 psychological impact in 3109 healthcare workers in spain: the PSIMCOV group. Psychological Medicine. Advance online publication. https://doi. org/10.1017/S0033291720001671
Santamaría, M.D., Etxebarria, N.O., Rodriguez, I.R., Albondiga-Mayor, J.J., \& Gorrochategui, M.P. (2020). Impacto psicológico del COVID-19 en una muestra de profesionales sanitarios españoles. Revista de Psiquiatría y Salud Mental. Advance online publication. https://doi.org/doi:10.1016/i. rpsm.2020.05.004

*Shacham, M., Hamama-Raz, Y., Kolerman, R., Mijiritsky, O., Ben-Ezra, M., \& Mijiritsky, E. (2020). COVID- 19 factors and psychological factors associated with elevated psychological distress among dentists and dental hygienists in Israel. International Journal of Environmental Research and Public Health, 17(8), 2900. https://doi.org/10.3390/ijerph 17082900

*Shechter, A., Diaz, F., Moise, N., Anstey, D.E., Ye, S., et al. (2020). Psychological distress, coping behaviors, and preferences for support among New York healthcare workers during the COVID-19 pandemic. General Hospital Psychiatry. $\quad$ https://doi.org/10.1016/i. genhosppsych.2020.06.007

*Shen, X., Zou, X., Zhong, X., Yan, J., \& Li, L. (2020). Psychological stress of ICU nurses in the time of COVID-19. BMC Critical Care, 24,200 https://dx.doi. org/10.1186\%2Fs13054-020-02926-2

Sun, L., Sun, Z., Wu, L., Zhu, Z., Zhang, F., Shang, Z., ... \& Liu, W. (2020a). Prevalence and risk factors of acute posttraumatic stress symptoms during the COVID-19 outbreak in Wuhan, China. MedRxiv. Advance online publication. https://doi. org/10.1101/2020.03.06.20032425

Sun, N., Wei, L., Shi, S., Jiao, D., Song, R., Ma, L., ... \& Wang, H. (2020). A qualitative study on the psychological experience of caregivers of COVID-19 patients. American Journal of Infection Control, 48(6), 592-598. https:// doi.org/10.1016/i. ajic.2020.03.018

Tan, B. Y., Chew, N. W., Lee, G. K., Jing, M., Goh, Y., Yeo, L. L., ... \& Sharma, V. K. (2020). Psychological impact of the COVID-19 pandemic on health care workers in Singapore. Annals of Internal Medicine, 173(4), 317-320. https://doi.org/10.7326/ M20-1083 
Vahedian-Azimi, A., Moayed, M. S., Rahimibashar, F., Shojaei, S., Ashtari, S., \& Pourhoseingholi, M. A. (2020). Compare the severity of psychological distress among four groups of Iranian society in COVID-19 pandemic. BMC Psychiatry. Advance online publication. https://doi.org/10.21203/ rs.3.rs-23828/v1

*Varshney, M., Parel, JT., Raizada, N., \& Sarin, SK. (2020). Initial psychological impact of COVID-19 and its correlates in Indian Community: An online (FEEL-COVID) survey. Plos One, 15(5), e0233874. https://doi. org/10.1371/journal.pone.0233874

Wang, J., Cheng, Y., Zhou, Z., Jiang, A., Guo, J., Chen, Z., \& Wan, Q. (2020). Psychological status of Wuhan medical staff in fighting against COVID-19. Medical Journal of Wuhan University. Advance online publication. https://dx.doi. org/1.14188/i.1671-8852.2020.0098

Wang, S., Xie, L., Xu, Y., Yu, S., Yao, B., \& Xiang, D. (2020). Sleep disturbances among medical workers during the outbreak of COVID-2019. Occupational Medicine, 70(5), 364-369. https://dx.doi. org/10.1093\%2Foccmed\%2Fkqaa074

Wu, W., Zhang, Y., Wang, P., Zhang, L., Wang, G., Lei, G., ... \& Luo, M. (2020). Psychological stress of medical staffs during outbreak of COVID-19 and adjustment strategy. Journal of Medical Virology, 92(10), 1962-1970. https://doi.org/10.1002/ imv.25914

*Wu, K., \& Wei, X. (2020). Analysis of Psychological and Sleep Status and Exercise Rehabilitation of Front-Line Clinical Staff in the Fight Against COVID-19 in China. Medical Science Monitor Basic Research, 26, e924085-1. https://dx.doi. org/10.12659\%2FMSMBR.924085

*Xiao, H., Zhang, Y., Kong, D., Li, S., \& Yang, N. (2020). The effects of social support on sleep quality of medical staff treating patients with coronavirus disease 2019 (COVID-19) in January and February 2020 in China. Medical Science Monitor: International Medical Journal of Experimental and Clinical
Research, 26, e923549-1. https://dx.doi. org/10.12659\%2FMSM.923549

$X_{u}$, J., Xu, QH., Wang, C.M., \& Wang, J. (2020). Psychological status of surgical staff during the COVID-1 9 outbreak. Psychiatry Research. Advance online publication. https://doi. org/10.1016/i. psychres.2020.112955

*Yuan, S., Liao, Z., Huang, H., Jiang, B., Zhang, X., et al. (2020). Comparison of the indicators of psychological stress in the population of Hubei Province and nonendemic provinces in China during two weeks during the coronavirus disease 2019 (COVID-19) outbreak in February 2020. Medical Science Monitor: International Medical Journal of Experimental and Clinical Research, 26, e923767-1. https://dx.doi. org/10.12659\%2FMSM.923767

*Zhang, C., Yang, L., Liu, S., Ma, S., Wang, Y., et al. (2020a). Survey of insomnia and related social psychological factors among medical staff involved in the 2019 novel coronavirus disease outbreak. Frontiers in Psychiatry, 11, 306. $\quad$ https://doi.org/10.3389/ fpsyt.2020.00306

*Zhang, WR., Wang, K., Yin, L., Zhao, WF., Xue, Q., et al. (2020b). Mental health and psychosocial problems of medical health workers during the COVID-19 epidemic in China. Psychotherapy and Psychosomatics, 89(4), 242-250. https:// doi.org/10.1159/000507639

*Zhu, Z., Xu, S., Wang, H., Liv, Z., Wu, J., et al. (2020a). COVID-19 in Wuhan: Immediate psychological impact on 5062 health workers. MedRxiv. https://doi. org/10.1101/2020.02.20.20025338

*Zhu, J., Sun, L., Zhang, L., Wang, H., Fan, A., et al. (2020b). Prevalence and influencing factors of anxiety and depression symptoms in the first-line medical staff fighting against COVID-19 in Gansu. Frontiers in Psychiatry, 11, 386. https://dx.doi. org/10.3389\%2Ffpsyt.2020.00386 